\title{
The Comparison of Routers by Firms Cisco, Juniper and Huawei
}

\author{
Sergey V. Makarov ${ }^{1}$, Vyacheslav V. Ostanin ${ }^{1} \&$ Ilia V. Vaitkov ${ }^{2}$ \\ ${ }^{1}$ Yurga Institute of Technology of National Research, Tomsk Polytechnic University, Kemerovo region, Yurga, \\ Russia \\ ${ }^{2}$ Siberian Federal University, Krasnoyarsk, Russia \\ Correspondence: Sergey V. Makarov, Yurga Institute of Technology of National Research, Tomsk Polytechnic \\ University, Kemerovo region, Yurga, Russia. E-mail: sergmakarov21@mail.ru
}

Received: September 10, 2014

Accepted: September 14, $2014 \quad$ Online Published: November 19, 2014

doi:10.5539/mas.v8n6p313

URL: http://dx.doi.org/10.5539/mas.v8n6p313

\begin{abstract}
According to data, published by Cisco Company a volume of internet traffic increases constantly and this tendency reserves further. That is why the output of routers stays still an important factor, which influences the quality of services rendered by internet access providers, satisfying clients' demands and, as a consequence, operators' profit. Contained in the article results of routers comparison by firms Cisco, Juniper and Huawei affords us getting into distinctions between products of these firms and place an emphasis on their significant peculiarities.
\end{abstract}

Keywords: router, internet, supported devices, carrying capacity, network core

\section{Introduction}

Router is called a specialized net computer, which streams packets of network layer (layer 3 of model OSI) between net segments, on the assumption of certain rules and network topology information. These devices are divided into hardware and software-control.

Routers provide load decrease on the net, dividing it on broadcast or collision domains and also filtering packets (Al-Kasassbeh and Adda, 2008). In general these devices are dedicated for various types of network connection, at times incompatible of record and systems application architecture. Also routers are used for access from local area network in the Internet, creating functions of firework and network addresses translation.

The principle of router work is in the following. A device gets listener address, mentioned in protocol packet and routing directory and finds data transmission path. If in routing directory for the address the described route is absent, this packet is ignored (routing directory contents information, on which basis the device take the decision concerning further packet forwarding).

Another way of route detecting is in packet forwarding, with usage of source address, upper-level protocols and other information, containing in packet headers of network layers. Also routers filter data transfer stream on the basis of certain rules for content advisor network listener address and source address translation, encryption and transmitted data, etc.

In its objectives routers are classified the following way:

a) Core routers, which put into practice company's network producing (central net may include certain network of area networks, which are placed in different buildings and use various computers, operating systems and network engineering);

b) Routers of regional brunches of organization connect them with central net and between themselves;

c) Routers of remote offices connect local nets of remote offices with central network or with network of regional divisions by using means of global connections and channels;

d) Routers of local nets are designed for splitting large local networks onto subnetworks.

Router is the device of network level of Open Systems Interconnection OSI, which uses one or more metric for optimal way of net traffic transmission on the basis of network level information. Thereof this definition follows that the router, first of all, is necessary for defining further data way, sent in a large and sophisticated network. The user of such network sends his data in the network and specifies an address of his requester. And that is all. 
The data go on through the network in points with connectivity forking come on the routers, which are exactly settled in such points. The router chooses the further and the better path. What path to choose is defined by volume indicators, which are called metrics (Al-Kasassbeh and Adda, 2009). The best path is a path with the smallest metrics. In the metrics few displays, for example, path length, transmission time etc. can be taken into account. Routers are divided into devices of upper, middle and lower classes. Upper class highend routers serve network interconnection of the enterprise. They preserve a set of protocols and interfaces, at that not only standard, but sometimes extremely exotic.

Routers for core networks and remote offices have various systems application architecture because different functional (Huh et al., 2006) and operational demands are put forward to them. Backbone routers must be expandable. Routers of local area networks units for which, as a rule, fixed port configuration is set, contain only one processor, which manages of functioning three or four interfaces.

The backbone router consists of the following main components: network interface card, which depend from protocols and serving as interfaces with local and global networks; control processing unit, defining the route and updating the information concerning topology of the main highway. After packet arrival to the transition module it analysis destination address and receives commands of control processing unit for output port diagnostics. After, the packet by router's highway is transferred to the interface module, which serves for connection with addressed segment of local or global network.

Route calculation of data transmission is created by software. Corresponding software tools are entitled of routing protocols. Reasonable capability of their functioning is based on routing algorithms. Routing algorithms calculate the delivery cost and chose the way with lower cost. Straightforward routing algorithms define the route on the basis of the lowest number of intermediate (transit) nodes on the way to destination station. More complex algorithms in terms of "cost" put few objectives a packet delay and communication bandwidth or money value of connection. Basic result of routing algorithm work is a creation and routing directory support wherein registered all routing information. Content of TOC depends on the used routing information protocol.

Plain requirements to routing algorithm are:

- Optimality of path selection;

- Overall simplicity practice;

- Resist ability;

- Fast convergence;

- Realization flexibility.

Optimality of route choice is the main parameter of the algorithm, that doesn't claim explanations. Routing algorithms should be simple in realization and they should use as few resources as possible. Algorithms must be persistent to equipment fault on the first-choice route, high loads and errors in network construction. Convergence is a negotiation process of network topology information between routers. If particular event in the net results in that some routes become unavailable or new routes arise, the routers send messages about it to each other networkwide. After receiving these messages the routers handle reassign optimal routes that in their turn can produce new thread of messages. This process must finish at that sufficiently quickly otherwise in network topology may arise or net in general may stop functioning at that sufficiently quickly, although in network topology may arise loops or the net may stop functioning at all. Routing algorithms must quickly and precisely take into account changes in network condition (for example nod or network segment fault). Advantages of flexible realization don't demand comments.

On this subject much attention is paid in the works:

1) J. Mu, W. Wang, B. Zhang, W. Song, An adaptive routing optimization and energy-balancing algorithm in ZigBee hierarchical networks, EURASIP Journal on Wireless Communications and Networking, 2014, art. no. 43.

2) Y. Shang, Mean commute time for random walks on hierarchical scale-free networks, Internet Mathematics, 2012, vol. 8, no. 4, 321--337.

3) T. P. Peixoto, Hierarchical block structures and high-resolution model selection in large networks, Physical Review X, 2014, vol. 4, art. no. 011047.

Routing algorithms may be:

- Statistic or dynamic; 
- One route or all route;

- One level or multilevel;

- Intra or cross-domain;

- One addressed or team.

For statistic (inadaptive) algorithms routes are chosen in advance and are typed in manually into routing directory, where the information concerning what port to send the packet with corresponding address is stored. Protocols, developed on the basis of statistic algorithms are called unroutable. The examples of unroutable protocols may serve LAT (Local AreaTransport, a transport protocol for channel areas) of DEC company, a connection data terminal protocol and NetBIOS. Usually bridges work with these protocols, because they don't distinguish network layer protocols.

With the usage of dynamic algorithms routing directory live with network topology change and traffic in it. Dynamic algorithms are distinguished by the way of getting the information concerning network condition, time of routing changing and the used appraisal ratio.

One route protocols define only one route. Not always it turns to be optimal. All route algorithms suppose few routes to one and the same acquirer (Kolbasinsky, 2013). Also algorithms afford to convey information by several channels at the same time, which means the increase of network throughput rate. Route algorithms may work in the nets with one level or hierarchical architecture. In one-level net all its fragments have the same priority, that, as a rule, is caused by commonality of their functional destination. Hierarchical network contains subnetworks (network fragments). Routers of upper level attach special part of the network, which is called backbone cabling (supporting part). Routers of backbone cabling transfer packages between lower level networks. Hierarchical structures in large and complicated networks allow simplifying significantly the process of network managing, makes easier isolation of network segments etc. For example logistics isolation of network segments allows security-edge gateway installations.

Some algorithms of routing function only as late as in limits of their domains (interdomain routing), and the others within their domains and contiguously-allocated with them (interdomain routing) (Thai and Wan, 2003). In that case a domain mean routing area, in which works one or few protocols. In different domains work different protocols. If the connection of domains is necessary, the interdomain routing is used. One address routing algorithms are supposed for transmission of concrete information (on one or few routes) to just one target (Fengxiang and Shunji, 2006). Multi-address (or group) algorithms are able to transmit information to many targets simultaneously. When the router receives a package it retrieves a destination address and identifies the route to which the package should be sent. Usually routers hold data about few possible routes. The route choice depends on few factors, which include:

- An applied system of route length calibration (its metric);

- High level routing protocol;

- Network topology.

The main task of packages transmission level is a packet switching of different users. General scheme of packages transmission is the following: one of possible relay nodes is chosen (this information comes from routing level on which it is calculated on the address of recipient), target data link level is formed and package sending is fulfilled. Besides, at this stage package fragmentation, checksum test etc. may be fulfilled. Routers (more precisely level of routing) work on the network level of reference model OSI. The level of packages advancement functions on the channel level. The work on the network level affords producing intellectual packaging processing. Due to routers general work with protocol IP, they must support a link without creating of logical connection between subscribers. With this each packaging is processed and sends to receiver independently. Manufacturers on creating routers use three general types of architecture:

- $\quad$ Single-processor;

- Intense single-processor;

- Symmetric multi-processor.

With uniprocessor design on central processor of router attaches all job on traffic processing: filtration and packages transfer, renewal of routing directory, detachment of service packs (Nazri and Zin, 2008), work with protocol SNMO, managing pack forming etc. This leads to that the router may become a bottleneck in the network on the loading increase. Even the usage of RISK processors doesn't solve the problem. For slope of 
enlightenment of such architecture an intense uniprocessor architecture is used. In functional scheme of the router son cards, which are in charge of fulfillment of these or those special tasks are chosen. Each such module of router is equipped with its (peripheral) processor. Herewith partial relief of central processor, which is in charge only for those tasks, which can not be entrusted to peripheral happens. In general and this architecture is not capable to solve all problems, connected with productivity. Symmetric multi-processor architecture is deprived listed disadvantages because erect load on all packages takes place.

\section{Methodology}

10-15 years earlier Cisco company was the only producer of net equipment, which is able to meet by its products the necessities of all users of global net Internet services (Solie and Lynch, 2004) from net providers to small companies, which have a couple of affiliates and which need the net, which will be able to unite all affiliates (www.nag.ru, 2014). According to some data, a segment of Cisco Company among producers of net equipment attained 80\% (www.cisco.com, 2014).

However, today firm Cisco has serious competitors, which are able to infringe hegemony of Cisco firm at the market of net equipment.

American company Juniper, established in year 1996 (www.juniper.net, 2014) represents as a serious competitor for Cisco in the net equipment producer sphere for internet-providers. To a large extent these corporations are alike, they have their own academies, preparing specialists for work with global net Internet, products of these companies have own operational systems. But there also present differences between named corporations, aroused, first of all, that Juniper, historically was oriented at development of decisions for decisions development for large corporations and providers of internet services, while Cisco is looking for embracing of all market (www.expressindia.com, 2014). Such difference in approaches found reflection as in produced products and in trend of specialists' preparation in Cisco and Juniper academies.

The third company, concerning which we'll talk in present article was not so long ago little known and by majority was perceived as something a level lower than such corporations as Cisco and Juniper. In majority of cases with comparison of production by Huawei with production of other companies (Cisco and Juniper, in particular), many people talked that given production will bring only extra worries and difficulties, which are possible to escape if to use production of notable competitors. As against Juniper corporation firm Huawei didn't set for only production for large corporations, which have many affiliates, but it chose the way of Cisco company, by making output for all sectors of internet services market. Huawei company became the first provider in the world, which run the system NE5000E to $2+8$ clusters in April 2008 and leading the internet to the era 10T (www.huawei.com, 2014). High capacity of the device corresponds the requirements of large-scale of service deployment of broad-band coupling for operators. NE5000E uses progressive non-blocking multi-put fabric. Contra directional zero error capacity of one block interface can be increased to $1.28 \mathrm{~TB}$ second. A view carries capacity in $40 \mathrm{~Gb} / \mathrm{sec}$ to AGP slot. Production of one-block communicating makes up 1600 megapixels per sec.

With innovation technology of hardware expansion in service cluster system NE5000E props up a regime of one block and regimes of multi-block clusters, such as back-to-back, $1+4$ and $2+8$. The design of future allows extension of cluster system to $16+64$ of multi-block configuration.

As far as firms have different directionality, Juniper, in particular doesn't produce products for small offices, that is why comparison of model range and strip from them, production alike on characteristics and solving problems, turns to be impossible, that is why models considered to be of carrier class more close in their characteristics.

\section{Results}

Cisco firm will be presented by router Cisco ASR 9922 (pic. 1a). Given product backs up capacity 11 Tbps (www.ciscoexpo.ru, 2014) and this way it is the most productive product of ASR series.

For comparison in given article firm Juniper presents a model Juniper MX2020 (figure 1b), which backs up carrying capacity from 34.4 to 80 Tbps.

Routers 3D Universal Edge MXseria from Juniper Networks present to network operators an optimization, high productivity, reliability and scalability, which are necessary for quickly developing market and technical environment.

Operation system Junos and a set of micro schemes Junos Trio make the routers 3D MX series the most programmable edge platform in the brunch. Besides, scalability of frequency pass band for subscribers and services provide protection of investments even at quickly developing markets.

MX series is optimized for company Juniper Networks specific strategy Software Defined Network (SDN), 
separating planes of distant control, management, services and redirecting. Subsystem JunosV App Engine provides virtualization of net services under the guidance of platform Junos Space Network Management.

Type spheres in which service suppliers can use routers MX series: support of Carrier Ethernet and VPN level 2 (Pang et al., 2003), prevalence of video, communications services and IPTV, aggregation for stationary and tandem mobile links networks, IP-routing and support of VPN level 3, functions of wide band media gateway, operated services, cloud computing, interfaces for data processing centers, closures for packed core and consolidation of services for nets of mobile links. Corporate application fields: consolidation and spiking in centers of data management, link on the net WAN and university nets.

A support of carrying capacity in $34.4 \mathrm{Tbps}$ in present moment, and the possibility of scaling up to $80 \mathrm{Tbps}$ in future, separates ready for use of software-defined networking edge router Juniper MX2020 with high production and density along the platforms of frontier routing in one pack, accessible in a branch for the present moment.

MX2020 supports a very high density $10 \mathrm{GbE}, 40 \mathrm{GbE}$ and $100 \mathrm{GbE}$, interfaces, and also obsolete methods of connection SONET/SDH, ATM and PDH. It also supports subscribers' management with wide-band connection, modern opportunities of time synchronizing and virtualization, which meet strict demands of mobile services. Due to scalable in industry-leading set of opportunities the router MX2020 ideally suited for boarders and consolidated borders and net core and application cores as well.

Router MX2020 is equipped with scalable and fully reserved architectures for provision of highest reliability levels. It divides general components of routing/control and commutation, port boards, service packages and applications with other platforms MX, providing investment protection and modernization for existing customers of MX series.

Huawei represents model Huawei NE5000E (figure 1c). This very model in 2008 afforded the firm Huawei show itself to be arch-rival in the area of Internet backbone.

Cluster router NetEngine 5000E (for short NE5000E) is a backbone router, which is used in internet backbones Internet, backbone city nets, Internet centres of data and basic Internet-networks NE5000E posses extra high speed of commutation and eminent performance characteristics of redirection due to processors Solar Series of civil production, due to progressive design of commutator's optical backbone connections, distributed and scalable platform of software. This device fully meets requirements of basic networks of next generation concerning operating factors, service quality and operation capabilities.

First complex solution 40G and 100G NE5000E carries 40G POS and 40G DWDM. Combining advantages of 40G IP and 40G of optical nets, company Huawei offers solution POS+OTN 40G with a row of scenarios and supports 40G DWDM, that fully satisfy demands of consumers to flexible nets.

Due to innovation asymmetric balance of loadings, the platform $40 \mathrm{G}$ provides asymmetric binding $40 \mathrm{G}$ to existing channels $10 \mathrm{G}$, what provides prominent protection of statement nesting. Due to thirst in the brunch of built-in TDCM intellectual interaction FEC and TDCM, Huawei solutions 40G regulate dynamically dispersion compensation and provide a really ready for usage nets 40G, maximally decrease the cost of network deployment of $40 \mathrm{G}$ and technical servicing. In the year 2009 NE5000E became the first in the world complex solution for $100 \mathrm{G}$ cluster systems.

NE5000E presents multi-level protection from the device to the network. For protection on the devices level, NE5000E contains passive plane where all key components of the device are switched in "hot-pluggable" and posses a function of hot back up. The solution contains nonstop address modification (NSF) on the basis of states and hot-swappable and nonstop routing (NSR) on the panel level and on the processes level in all scenario.

As the result, the system NE5000E reaches $99.999 \%$ of reliability of carrier-class. The system NE5000E is initially designed with taking into account the concept of ecological cleanness. Processor's core NE5000E, with the usage of technology $65 \mathrm{~nm}$, decreases energy consumption on 30\%. The usage of heat dissipation system at the expense of air cycle in USSR is significantly higher than heat dissipation, and this way it decreases energy consumption to heat dissipation on 50\%. The system applies conjugated optical fiber conjugations (OFC), which can be configured according to necessity. Compact design of a block allows sizes decreasing and the weight of device and it doesn't claim modifications of control rooms. The design, expansion and exploitation of cluster system NE5000E is ecologically safe.

Equipped with distributed forward hardware and non-blocking switching technology, NE5000E posses an operator class of reliability, capability to high speed transmission, high processability of bandwidth and an excellent zoom feature, due to which this system will be able to satisfy demands of non-stop increasing services 
in data transmission and video on backbones areas.

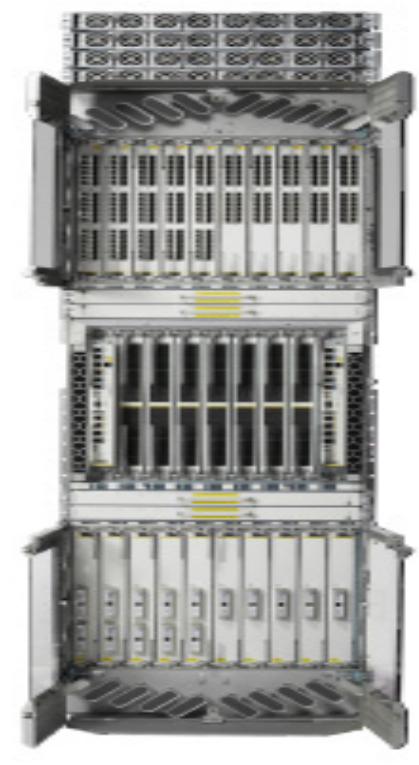

a

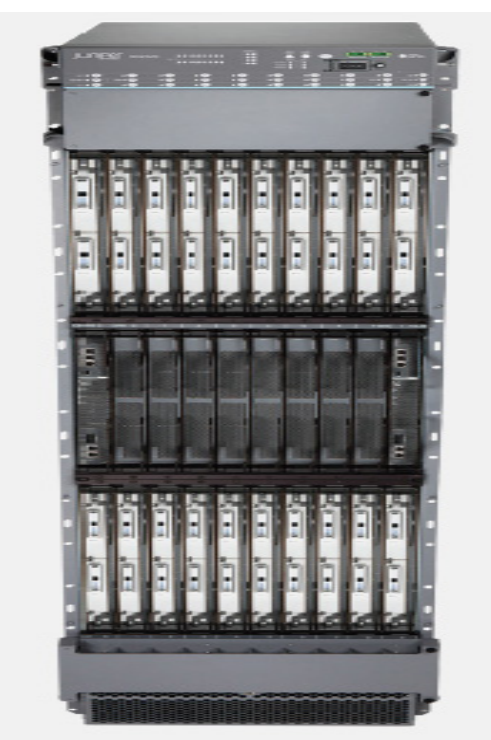

b

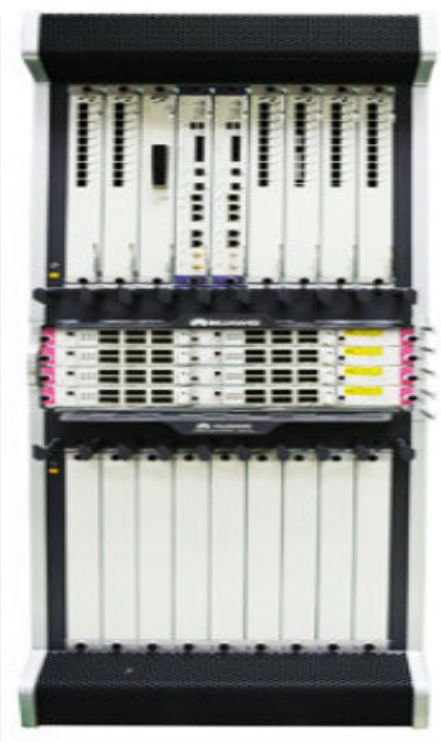

c

Figure 1. Routers of Cisco, Juniper and Huawei companies: a) Cisco ASR 9922; b) Juniper MX2020; c) Huawei NE5000E

Table 1. Comparison of routers of firms Cisco, Juniper, Huawei

\begin{tabular}{|c|c|c|c|}
\hline Compared indexes & Cisco ASR 9922 & Juniper MX2020 & Huawei NE5000E \\
\hline System capacity & Up to $11 \mathrm{Tbps}$ & Up to $80 \mathrm{Tbps}$ & Up to $6.4 \mathrm{Tbps}$ \\
\hline Slots quantity & 20 & 20 & 16 \\
\hline Slot capacity & $550 \mathrm{~Gb} / \mathrm{s}$ & 2 Tbps & $400 \mathrm{~Gb} / \mathrm{s}$ \\
\hline Processor & $\begin{array}{l}2.27 \mathrm{GHz} \\
\text { Quad Core }\end{array}$ & 1.8 GHz QuadCore & $\begin{array}{l}1.5 \mathrm{GHz} \\
\text { QuadCore }\end{array}$ \\
\hline Main storage volume & Up to $12 \mathrm{~GB}$ & $16 \mathrm{~GB}$ & Up to $16 \mathrm{~GB}$ \\
\hline Operation system & Cisco IOS & JunOS & VRP \\
\hline $\begin{array}{l}\text { Dimensional specifications } \\
\text { (Highxwidthxdepth), cm }\end{array}$ & $191 \times 45 \times 73$ & $200 \times 44 \times 92$ & $124 \times 44 \times 80$ \\
\hline Matrix & Dedicated Card & Dedicated Card & Dedicated Card \\
\hline $\begin{array}{l}\text { Support for optical } \\
\text { interfaces } 40 \mathrm{Ge}, 100 \mathrm{Ge}\end{array}$ & Yes & Yes & Yes \\
\hline $\begin{array}{l}\text { Operating temperature, } \\
\text { degrees C }\end{array}$ & -5 to +55 & 0 to +40 & 0 to +40 \\
\hline Mass, kg & 471 & 680 & 300 \\
\hline
\end{tabular}

\section{Conclusion and Discussion}

As it already mentioned at the beginning of the article, by many specialists production of Huawei company is perceived as something really yielding the market leaders (Juniper and Cisco), as it is seen from table, router NE5000E really turned out on the last place concerning on acceptance rate as general acceptance rate the same as acceptance rate of each separate slot. But, on the other hand, the representative of Huawei company possess the smallest dimensions and is developed for functioning as a part of a cluster, that someway highlights is in the competitor's list.

Concerning the products of firms Cisco and Juniper, coming to conclusion that in present comparison Juniper MX2020 turned out to be way ahead of its competitors. And, although Cisco ASR 9922 has the same quantity of slots as his competitor, it is strongly looses by comparison of slots capacity that is why it loses to Juniper MX2020 in general capacity. But you should remember, that net quality doesn't determined by productivity of 
network core, in the net may get along devices of different manufacturers and different productivity, the net may be built with the help of over-the-air or wired net of data communication, there exist many kinds of wired or wireless data communication. And each of these nuances influences the data transfer rate for a final consumer.

In conclusion of our analysis of the router showed, that they possess doubtless advantages. Routers don't bring any restrictions in net topology.

In frames of further researches the plan is to make different tests in-situ on the described in the paper equipment, because declared by manufacturers characteristics frequently differ from those, which we obtain on the run of normal job at the equipment.

\section{References}

Adhami, M., \& Halavati, I. (2013). Simulating and Implementing a Proposed IP Multicasting Service in the Network of Isfahan University Based on IGMP-AC Multicast System. Life Science Journal, 10(7s).

Al-Kasassbeh, M., \& Adda, M. (2008). Analysis of mobile agents in network fault management. Journal of Network and Computer Applications, 31, 699-711. http://dx.doi.org/10.1016/j.jnca.2007.11.005

Al-Kasassbeh, M., \& Adda, M. (2009). Network fault detection with Wiener filter-based agent. Journal of Network and Computer Applications, 4(32). http://dx.doi.org/10.1016/j.jnca.2009.02.001

Fengxiang, Z., \& Shunji, A. B. E. (2006). A DoS/DDoS Attacks Detection Scheme Based on In/Out Traffic Proportion. Information and Communication Engineers, 105, 7-11. ISSN: 0913-5685.

Huh, I., Lee, J. Y., \& Kim, B. C. (2006). Decision of Maximum Congestion Window size for TCP performance Improvement by Bandwidth and RTT measurement in Wireless Multi-hop Networks. International Journal of Information Processing Systems.

$\mathrm{Mu}$, J., Wang, W., Zhang, B., \& Song, W. (2014). An adaptive routing optimization and energy-balancing algorithm in ZigBee hierarchical networks. EURASIP Journal on Wireless Communications and Networking, 2014(43). http://dx.doi.org/10.1186/1687-1499-2014-43

Kolbasinsky, D. B. (2013). Information Networks and TV Communications, pp. 431.

MX2020 and 2010 3D UNIVERSAL EDGE ROUTERS. Retrieved August 31, 2014, from http://www.juniper.net/us/en/local/pdf/datasheets/1000417-en.pdf

Nazri, M. I., \& Zin, A. M. (2008). Measurement and Characterization of Network Traffic Utilization between Real Network and Simulation Modeling in Heterogeneous Environment. Int. J. of Comp Sc and Net Sec [IJCSN], 8(3), 326-337.

Nazri, M. I., \& Zin, A. M. (2008). Emulation Network Analyzer Development for Campus Environment and Comparison between OPNET Application and Hardware Network Analyzer. European Journal of Scientific Research, EJSR, 24(2), 270-291.

Pang, Q., Liew, S. C., Fu, C. P., Wang, W., \& Li, V. O. (2003). Performance study of TCP Veno over WLAN and RED router. IEEE Global Telecommunications Conference - GLOBECOM'03, 6, 3237-3241. http://dx.doi.org/10.1109/GLOCOM.2003.1258834

Solie, K., \& Lynch, L. (2004). CCIE Practical Studies (1st ed). Cisco Press.

Peixoto, T. P. (2014). Hierarchical block structures and high-resolution model selection in large networks. Physical Review X, 4, 011047. http://dx.doi.org/10.1103/PhysRevX.4.011047

Thai, B. R., Seneviratne, W. A., \& Rakotoarivelo, T. (2003). Integrated personal mobility architecture: A complete personal mobility solution. Mobile Networks and Applications, 8(1), 27-36. http://dx.doi.org/10.1023/A:1021115610456

Shang, Y. (2012). Mean commute time for random walks on hierarchical scale-free networks. Internet Mathematics, 8(4), 321-337. http://dx.doi.org/10.1080/15427951.2012.685685

\section{Web References}

Architecture of Platform Cisco ASR 9000. Date Views 31.08.2014 Retrieved from http://ciscoexpo.ru/expo2012/sites/default/files/PDFDone/day1/SolutionsForTelecomOperators/14_15_15_ 15_PlatformArchitectureCiscoASR9000_agolovanov.pdf

Cisco Application Analysis Solution ACE Tutorials and Examples, Vol. Release 1.0, Corporate Headquarters, 170 West Tasman Drive: San Jose, CA 95134-1706 USA Cisco Systems, Inc. 
Cisco replaces Microsoft as world's most valuable company. Date Views 31.08.2014 Retrieved from $\mathrm{http}: / /$ expressindia.indianexpress.com/ie/daily/20000326/ibu26043.html

Cisco Visual Networking Index: Global Mobile Data Traffic Forecast Update, 2013-2018. Date Views 31.08.2014 Retrieved from http://www.cisco.com/c/en/us/solutions/collateral/service-provider/visual-networking-index-vni/white_pape r_c11-520862.html

Huawei Data Communication. Date Views 31.08.2014 Retrieved from http://www.huawei.com/en/products/data-communication/index.htm

Huawei's 400G Core Router Wins 53 Customers Worldwide. Date Views 31.08.2014 http://huawei.com/ilink/en/about-huawei/newsroom/press-release/HW_323863

Infonetics Research IP-routers market and Connection switches in sq. 3, 2013. Date Views 31.08.2014 Retrieved from

http://nag.ru/news/newsline/24329/infonetics-research-o-ryinke-ip-marshrutizatorov-i-kommutatorov-v-3-k v-2013-g.html

Juniper Networks. Date Views 31.08.2014 http://www.juniper.net/us/en/

\section{Copyrights}

Copyright for this article is retained by the author(s), with first publication rights granted to the journal.

This is an open-access article distributed under the terms and conditions of the Creative Commons Attribution license (http://creativecommons.org/licenses/by/3.0/). 